\title{
Nanotherapy in Advanced Prostate Cancer: Letter to the Editor
}

\author{
Gilles Plourde* \\ Department of Clinical Pharmacology and Physiology, University of Montreal, Canada
}

Submission: February 24, 2018; Published: March 13, 2018

*Corresponding author: Gilles Plourde, Associate Professor Department of Clinical Pharmacology and Physiology, Faculty of Medicine, University of Montreal, Montreal, Quebec, Canada, Email: gilles.plourde@hc-sc.gc.ca; drgplourde@gmail.com

Keywords: Advanced Prostate Cancer; Nanotherapy; Nanotechnology; Bone metastasis; Nano particles; Pgold nano particles; Lipid nano particle siRNA systems: the ranostic nano particles; Nano particulate-based medicine; Inorganic nano particles; Interleukin-8 (IL-8); Multifunctional Envelope-Type siRNA Delivery Nano particle; Anti-CD24 nano-targeted delivery and Prostate-specific membrane antigen (PSMA)-Targeted Theranostic Nano carrier

\section{Introduction}

Prostate cancer continues to be the second most common cause of cancer-related deaths in men. Recently, several new treatments have led to significant improvement in overall survival. These include the novel androgen receptor pathway inhibitors abiraterone acetate (Zytiga taken in combination with prednisone) and enzalutamide (Xtandi), the chemotherapy drugs taxanes, docetaxel (Taxotere) and cabazitaxel (Jevtana), and a bone targeting alpha-emitting radionuclide, radium-223 chloride (Xofigo) [1]. However, resistance and recurrence still persists, which limits patient benefit [2]. Prostate cancer stem cells (PCSCs) are resistant to most standard therapies, and are considered to be a major mechanism of cancer metastasis and recurrence [2].

Recently, nanotechnology opens a new era for drug delivery in cancer therapy. Nanotechnology is the engineering and manufacturing of materials from 1 to 100 nanometers in size [3-5]. Nanotechnology has been widely used in cancer diagnosis and therapy such as molecular imaging, molecular diagnosis, and targeting therapy. For example, nano vectors are used in clinical practice to help the targeted delivery of imaging contrast agents for diagnosis and anticancer drugs for treatment [3-5].

Recent advancements in cancer nanotechnology have facilitated a better way to diagnosis and provide therapy for prostate cancer. Nanotechnology has the potential to fight tumors at the site where it is located [3-5]. To achieve this purpose, there is a need to improve the therapeutic availabilities and the effectiveness of treatment for prostate cancer. Many therapeutic nano particles (NPs) have been developed with nanotechnology that can specifically target and deliver a variety of agents including chemo drugs to destruct, for example, the prostate cancer cells without causing any damage to the healthy cells [3-5]. For instance, theranostic (personalized medicine) NPs that have been developed to specifically target the prostate cancer cells are using targeting ligands and releasing the anticancer agents in a controlled and time-dependent manner in combination with assisted imaging to monitor the effectiveness of the therapy in real time [3-5]. Below, I will discuss some of the nano therapy approaches that are currently in clinical development that will be of interests for our patients and his primary care providers.

Bone metastatic castration-resistant prostate cancer (mCRPC) treatments remain a major challenge despite the high number of newer drugs being developed and authorised in recent years [1]. The major challenge for improving treatment outcomes for bone in mCRPC originates from the fact that the drugs are unable to reach the bone microenvironment and therefore are not able to induce the desired response [6]. These metastatic lesions are difficult to treat and often result in off target cytotoxicity from cancer treatments.

Gdowski AS 2017 and his colleagues have recently engineered a new bone-targeted NP that delivers the chemotherapy drug such as cabazitaxel (Jevtana) directly to the bone [6,7]. The NP formulation bind to the chemical structure of the bone and was effective at reducing tumour size, maintaining bone structure, and decreasing bone pain [6,7]. In these studies bone tumors were established for one week in mice (starting $n=6$ per group) then treated weekly with saline, free cabazitaxel, non-targeted NPs, or targeted NPs [6,7]. 
The targeted NPs had a strong pick release of cabazitaxel within the first 8 hours and a sustained release for up to 72 hours. The targeted NPs also had a fourfold increase in binding to bone at six hours and an eightfold increase at 72 hours when compared to the non-targeted NPs [6]. Mice treated with targeted NPs had no bone lesions on x-ray, with 100 percent in the saline and cabazitaxel groups and 33 percent in the non-targeted NP group with bone lesions. These studies also demonstrated a reduction in bone pain for the targeted NP group [6,7]. Gdowski AS et al. (2017) and his colleagues concluded that these targeted NPs work well to decrease tumor size and that they were able to maintain the bone structure and reduce bone pain, which is an ongoing challenge when treating advanced prostate cancer patients [6,7]. Even though, these results are very promising, this study is at a very early stage of clinical development and is not applicable to humans, at this time, but the clinical development is ongoing.

Globally, the use of nanotechnology in cancer treatment offers some exciting possibilities, including the possibility of destroying cancer tumors with minimal damage to healthy tissue and organs, as well as the detection and elimination of cancer cells before they form tumors [3-5]. Therefore, the effort to make these treatment approaches a reality for human patients is highly promising with further clinical development. For example, The Alliance for Nanotechnology in Cancer (https:// www.cancer.gov/sites/nano) established by the U.S. National Cancer Institute, is providing innovation and collaboration among researchers to resolve some of the major challenges in the application of nanotechnology to cancer [3-5]. In addition, there are many universities and pharmaceutical companies' worldwide that are currently working in this area. It is, therefore, possible that we will have nanotechnology treatment available for our patient with the next few years.

In this letter to the editor, with the use of a case report, I will summarize some of the main components associated with nanotherapy in order to be able to answer the questions raised by many patients and primary care providers dealing with patients suffering from mCRPC where treatment options become limited. The next section provides examples of the research that are currently underway; some of them are only at the preclinical development while others are at the clinical trial stage of clinical development. Obviously, in the context of this letter to the editor, only limited aspects of nanotherapy and its transport system will be discussed.

\section{Case Report}

The same 61 year-old patient known for an undifferentiated and an invasive stage IV mCRPC that was discussed in previous letters to the editor $[8,9]$ came to your office. He is currently treated with Zoladex, Zytiga and Prednisone. The bone pain has slightly decreased since he is taking Zytiga as well as Xgeva for bone metastasis. Since he is afraid about becoming resistant to these treatments, he wants to know what should be the best treatment approach for him when he will no longer be responsive to this second-line hormone therapy. He was looking at the medical literature and from what he has read; it seems that nanotherapy may be a promising option for him at middle and long-term.

Among the following items, which one(s) is/are considered the main elements involved in the comprehension of nanotherapy that you should discuss with your patients. Again, in the context of this letter, it is impossible to discuss all the elements associated with nanotherapy, rather the purpose here is to provide to the patients the information that is useful for an adequate counselling on the nanotherapy approach.

a. The use of nano particles to deliver drugs to cancer cells;

b. Photosensitizing agents;

c. Gold nano particles;

d. Lipid nano particle siRNA systems:

e. Theranostic nano particles;

f. Nano particles-based medicine:

g. In organic nano particles:

h. Interleukines-8 (IL-8):

i. Multifunctional Envelope-Type siRNA Delivery Nano particle:

j. Anti-CD24 nano-targeted delivery:

k. Prostate-specific membrane antigen (PSMA)-Targeted Theranostic Nano carrier.

1. All of the above should be considered for the counselling.

\section{Response: L}

i. The use of nano particles to deliver drugs to cancer cells: Perhaps the most known use of nanotechnology in drug delivery that is progressing in its clinical development is the use of NPs to deliver drugs to cancer cells [3-5]. Particles are engineered in a way that they can be attracted by diseased cells, which permit a localised treatment in those cells. Interestingly, this technique reduces damage to healthy cells in the body. Researchers are also continuing to look for more effective methods to target NPs carrying therapeutic drugs directly to diseased cells. For example scientists have demonstrated an increased levels of drugs delivery to tumors by using two types of NPs. [3-5] The first type of NP locates the cancer tumor and the second type of NP (carrying the therapeutic drugs) locally from the signal generated by the first type of NP [3-5]. This approach can certainly be useful for our patient, but further studies are required before this approach can be used in clinical practice since it is only at an early stage of clinical development. Therefore, $\mathrm{A}$ is a good answer. 
ii. Photosensitizing agents: These agents enhance the ability of drug carrying NPs to enter the tumors [4-10]. First they let the photosensitizing agent accumulate in the tumor, then illuminate the tumor with infrared light. The photosensitizing agent causes the blood vessels in the tumor to be more porous, therefore more drug carrying NPs can enter the tumor [4-10]. This is also a promising approach for our patient pending further clinical development. Therefore, B is a good answer.

iii. Gold nano particles: Gold NPs that has already been used to deliver platinum to cancer tumors may reduce the side effects of platinum cancer therapy [3-5]. The key is that the toxicity level of platinum depends upon the molecule it is bonded to (the toxicity depends upon the oxidation state of the platinum). Therefore, the researchers chose a platinum containing molecule that has low toxicity to attach to the gold NPs. When the platinum bearing NP reaches a cancer, the tumor it encounters is exposed to an acidic solution which changes the platinum to its toxic state, in which it can kill cancer cells [3-5]. Another example of gold NPs is a method being developed to fight skin cancer using gold NPs to which RNA molecules are attached. The NPs are in an ointment that is applied to the skin. The NPs penetrate the skin and the RNA attaches to a cancer related gene stop the gene from generating proteins that are used in the growth of skin cancer tumors [3-5]. Recently, researchers from the University of Missouri-Columbia have found that gold NPs can treat prostate cancer in animals with fewer side effects than chemotherapy [11], which is highly promising for our patient pending further clinical development. Therefore $\mathrm{C}$ is a good answer.

iv. Lipid nano particle: siRNA systems: The androgen receptor plays a critical role in the progression of prostate cancer [1]. Silencing this protein using short-hairpin RNA (siRNA) has been correlated with tumor growth inhibition and decreases in serum prostate specific antigen (PSA) [12]. In a study, Lee JB, Zhang K, YI, Y et al. (2012) have investigated the ability of lipid nano particle (LNP) formulations of small-interfering RNA (siRNA) to silence androgen receptor in human prostate tumor cell lines in vitro and in LNCaP xenograft tumors following intravenous injection [12]. They found in In vitro screening studies using a panel of cationic lipids showed that LNPs containing a ionisable cationic lipid 2,2-dilinoleyl-4-(2-dimethylaminoethyl)-[1,3]dioxolane (DLin-KC2-DMA) exhibited the most potent androgen receptor silencing effects in LNCaP cells [12]. This is attributed to the ability of DLin-KC2-DMA-containing LNP to be taken up into cells and to release the siRNA into the cell cytoplasm following endocytotic uptake. DLin-KC2-DMA LNPs were also effective in silencing the androgen receptor in a wild-type androgen receptor expressing cell line, LAPC-4, and a variant androgen receptor expressing cell line, CWR22Rv1. Importantly, these authors demonstrated that LNP androgen receptorsiRNA systems containing DLin-KC2-DMA can silence androgen receptor gene expression in distal $\mathrm{LNCaP}$ xenograft tumors and decrease serum PSA levels following intravenous injection [12]. This is one of the first reports demonstrating the feasibility of
LNP delivery of siRNA for silencing androgen receptor gene expression in vivo [12]. This approach is very promising for patients having an advanced prostate cancer and seems more advanced in his clinical development that the other approaches discussed above. Therefore D is a good answer.

v. Theranostic NPs: Recent advancements in cancer nanotechnology have facilitated a better way to diagnosis and provide therapy for prostate cancer [1]. Nanotechnology has the potential to battle tumors at the site, where the cancer begins [3-5]. However, there is a need to improve the therapeutic availabilities and the effectiveness of conventional chemotherapeutic agents for prostate cancer. Many therapeutic NPs have been developed with nanotechnology that can specifically target and deliver variety of agents including chemo drugs to destruct the prostate cancer cells without causing any damage to the healthy cells [3-5]. Theranostic NPs have been developed to specifically target the prostate cancer cells using targeting ligands to release the anticancer agents in a controlled and time-dependent manner for cancer therapy in combination with assisted imaging to monitor the effectiveness of the therapy in real time [13]. The surface-modified polymers and metallic NPs have evolved as promising nanomaterials for targeted prostate cancer treatment and therefore is promising for our patient [3-5,13]. Therefore $\mathrm{E}$ is a correct answer.

vi. Nanoparticles-based medicine: We can define nano medicines as therapeutic or imaging agents which comprise a nano particle in order to control the bio distribution, enhance the efficacy, or otherwise reduce toxicity of a drug or biologic [3-5]. Bobo D 2016 have identified 51 FDA-approved nano medicines that met this definition and 77 products in clinical trials, with $\sim 40 \%$ of trials listed in clinical trials.gov started. While FDA approved materials are heavily weighted to polymeric, liposomal, and nano crystal formulations, there is a trend towards the development of more complex materials comprising micelles, protein-based NPs, and also the emergence of a variety of inorganic and metallic particles in clinical trials [14]. Bobo D 2016, concluded with some comments on future perspectives for nano medicines, which they expect to include more actively-targeted materials, multi-functional materials («theranostics») and more complicated materials that blur the boundaries of traditional material categories. A key challenge for researchers, industry, and regulators is how to classify new materials and what additional testing (e.g. safety and toxicity) is required before products become available [3-5]. Even with these challenges, the nano particulate medicine is highly promising considering the number of product currently approved and the others that are in clinical development, which means that it is not too speculative to expect the development of nano particulate that will be specific for the treatment of advanced prostate cancer soon. Therefore $\mathrm{F}$ is a good answer.

vii. Inorganic nano particles: Inorganic NPs are the potentially capable contender in the area of cancer therapy [35].They can effectively rectify and replace the drawbacks of the 
old conventional chemotherapy [15]. NPs possess a broad range of use in both diagnosis and imaging study of the cancer cells. So far researchers have done a large number ofworks denoting the effectiveness of inorganic NPs in cancer therapy [3-5]. Furthermore, the toxicity of inorganic NPs is the only concern at higher concentration, but utilization of biologically material or sources for the synthesized NPs and coating them with degradable polymers will reduce the risk of toxicity according to Pugazhendhi A 2018. Overall inorganic NPs are effective option for cancer therapy and diagnosis. Further they are overcoming the overall drawbacks of NPs which permit to define them as a potential remedy for future generation fighting against cancer worldwide. Therefore $\mathrm{G}$ is a good answer.

viii. Interleukin-8 (IL-8): IL-8 is a pro-angiogenic cytokine associated with advanced prostate cancer [16]. Aalinkeel R 2016, detected high levels of IL-8 in sera from patients with advanced prostate cancer compared with healthy controls and even in comparison with patients with benign prostatic hypertrophy [16]. This study has examined the role of IL-8 in the pathogenesis of metastatic prostate cancer. Aalinkeel $\mathrm{R}$ 2016, have developed a biocompatible, cationic polylactide (CPLA) nano carrier to complex with and efficiently deliver IL-8 small interfering RNA (siRNA) to reach prostate cancer cells in vitro and in vivo [16]. CPLA IL-8 siRNA nano complexes (nanoplexes) protect siRNA from rapid degradation, are nontoxic, have a prolonged lifetime in circulation, and their net positive charge facilitates penetration of cell membranes and subsequent intracellular trafficking [16]. The administration of CPLA IL-8 siRNA nanoplexes to immuno deficient mice bearing human advanced prostate cancer tumors produced significant antitumor activities with no adverse effects [16]. Systemic (intravenous) or local intra-tumor administration of IL-8 siRNA nanoplexes resulted in significant inhibition of advanced prostate cancer growth according to Aalinkeel R, et al (2016). Then with magnetic resonance imaging and ultrasonography of experimental animals they were able to demonstratea reduction of tumour perfusion in vivo following nanoplex treatment. This study demonstrated the efficacy of IL-8 siRNA nanotherapy for treatment-resistant human advanced prostate cancer which is also promising for our patient. Therefore $\mathrm{H}$ is a good answer.

ix. Multifunctional Envelope: Type siRNA Delivery Nano particle: With the capability of specific silencing of target gene expression, RNA interference (RNAi) technology is emerging as a promising therapeutic modality for the treatment of cancer and other diseases [3-5]. The main challenge for the clinical applications of RNAi is the safe and effective delivery of RNAi agents such as small interfering RNA (siRNA) to a tumor and cell type with sufficient cytosolic transport [3-5]. In a recent study, XuX, et al. (2017) proposed a multifunctional envelope-type NP platform for prostate cancer-specific in-vivo siRNA delivery. In this study many oligoarginine-functionalized and sharp pH-responsive polymers were synthesized and used for selfassembly with siRNA into NPs with the characteristic of longterm blood circulation and $\mathrm{pH}$-triggered oligoarginine-mediated endosomal membrane penetration [17]. By further modification with ACUPA, a small molecular ligand specifically recognizing prostate-specific membrane antigen (PSMA) receptor; this envelope-type nano platform with multifunctional properties can efficiently target PSMA-expressing prostate cancer cells and silence target gene expression. The systemic delivery of the siRNA NPs can efficiently silence the expression of prohibition 1 (PHB1), which is up regulated in prostate cancer and other cancers, and significantly inhibit prostate cancer tumor growth according to XuX, et al. (2017) [15]. These results suggest this multifunctional envelope-type nano platform could become an effective tool for prostate cancer-specific therapy and therefore for our patient. Therefore it is a good answer.

x. Anti-CD24 nano-targeted delivery: NP-mediated, noninvasively targeted and image-guided therapies have the potential to improve efficacy and safety of cancer therapeutics [3-5]. A recent study by Bharali DJ, et al (2017) reported the synthesis and the use of poly (lactide-co-glycolide)-polyethylene glycol (PLGA-PEG) NPs for targeted delivery of docetaxel in prostate cancer [18]. They synthesized docetaxel encapsulated NPs conjugated to anti-CD24 (Anti-body for targeting) and/or an optical probe (for tracking) and evaluated efficacy in a prostate cancer mouse model [18]. Bharali DJ 2017, observed preferential accumulation of anti-CD24 conjugated NPs (encapsulating docetaxel) compared to the non-conjugated NPs 24 hours after a single injection into luciferase-expressing PC3M (cell line) prostate cancer tumor. In the same mouse model, the same authors found significant $(\mathrm{P}<0.01)$ accumulation of docetaxel ( $\sim 10$-fold higher) in tumor after treatment with PLGA-PEG NPs encapsulating docetaxel and conjugated to anti-CD24 compared to non-conjugated NPs. The enhanced accumulation was associated with reduced tumor mass and tumor viability [18]. These data support the potential impact of CD24 conjugated NP in the delivery of chemotherapy in enhancing the differential tumor delivery and anticancer efficacy in prostate cancer. Therefore $\mathrm{J}$ is a good answer.

xi. Prostate-specific membrane antigen (PSMA): Targeted Theranostic Nano carrier: In a recent study by Flores 0 2017, reported that the use of a theranostic nano carrier (FolateHBPE (CT20p)) was capable of delivering a therapeutic peptide to prostate cancer tumors that express PSMA (folate hydrolase 1). This therapeutic peptide (CT20p) targets and inhibits the chaperon in containing TCP-1 (CCT) protein-folding complex, is selectively cytotoxic to cancer cells, and is non-toxic to normal tissue [19]. With the delivery of CT20p to prostate cancer cells via PSMA, a dual level of cancer specificity is achieved: (i) selective targeting to PSMA-expressing prostate tumors; and (ii) specific cytotoxicity to cancer cells with minimal toxicity to normal cells [19]. The PSMA-targeting theranostic nano carrier can image PSMA-expressing cells and tumors when a near infrared dye is used as transporter. Meanwhile, it can be used to treat PSMA-expressing tumors when a therapeutic, such as the CT20p peptide, is encapsulated within the nano carrier. Even when these PSMA-targeting nano carriers are taken up 
by macrophages, minimal cell death is observed in these cells, in contrast with doxorubicin-based therapeutics that result in significant macrophage death according to Flores 0, et al. (2017) [19]. Incubation of PSMA-expressing prostate cancer cells with the Folate-HBPE-(CT20p) nano carriers induces considerable changes in cell morphology, reduction in the levels of integrin $\beta 1$, and lower cell adhesion, eventually resulting in cell death. These results are relevant as integrin $\beta 1$ plays a key role in prostate cancer invasion and metastatic potential [19]. In addition, the use of the developed PSMA-targeting nano carrier facilitates the selective in vivo delivery of CT20p to PSMA-positive tumor, inducing significant reduction in tumor size. Therefore $\mathrm{K}$ is a good answer.

\section{Conclusion}

It is anticipated pending further clinical development that nanotherapy will play an important role in cancer therapy in the near future as part of a multimodality treatment, in combination with, or following other forms of cancer therapy, such as hormonal therapy as in our patient. For our patient we can be reassuring that nanotherapy represents an interesting option for him when he will become resistant to the second-line hormone therapy (Zytiga). However, the approaches discuss above clearly demonstrated the different level of clinical development where some approaches will be available sooner than others.

Since our patient is still responding very well on second-line hormone therapy, we can still hope that after our patient will become resistant to Zytiga, nano Therapy will be at a higher level of clinical development and will be available for him either as an authorised Health Product or in the context of clinical researches or through the Special Access Program.

\section{References}

1. Plourde G Advanced prostate Cancer: A case Report. Elsevier, Netherlands.

2. Wei Qin, Yongjiang Zheng, Bin-Zhi Qian, Meng Zhao (2017) Prostate Cancer Stem Cells and Nanotechnology: A Focus on Wnt Signaling. Front Pharmacol 8: 153.

3. Vinoth Kumar Lakshmanan (2016) Therapeutic efficacy of nanomedicines forprostate cancer: An update. Investig Clin Urol 57(1): 21-29.
4. Nanotechnolog in Cancer Treatment (2017) understanding nano.com.

5. (2017) Nano-targeting treatment for prostate cancer. Nano technology general news.

6. Gdowski AS, Ranjan AP, Sarker MR, Vishwanatha JK (2017) Efficient Bone Microenvironment Nano-Targeting for Improved Therapy for Bone Metastatic Prostate Cancer.

7. Gdowski AS, Ranjan A, Sarker MR, Vishwanatha JK (2017) Bonetargeted cabazitaxel nanoparticles for metastatic prostate cancer skeletal lesions and pain. Nanomedicine (Lond) 12(7): 2083-2095.

8. Plourde G (2017) Gene Therapy in Advanced Prostate Cancer: Letter to the Editor. J of Pharmacol \& Clin Res 4(1): 555627.

9. Plourde G (2017) Immunotherapy in Advanced Prostate. J of Pharmacol \& Clin Res 4(2).

10. Wei Wang, Guihai Chen, Yongping Chen (2013) Nanotechnology as a Platform for Thermal Therapy of Prostate Cancer. Journal of Molecular Biomarkers \& Diagnosis 4: 2.

11. (2016) Gold nanoparticles could treat prostate cancer with fewer side effects than chemotherapy.

12. Lee JB, Zhang K, YI Y (2012) Lipid nanoparticle sirna systems for silencing the androgen receptor in human prostate cancer in vivo. International Journal of Cancer 131(5): E781-E790.

13. Cherian AM, Nair SV, Lakshmanan VK (2014) The role of nanotechnology in prostate cancer theranostic applications. J Nanosci Nanotechnol 14: 841-852.

14. Bobo D, Robinson KJ, Islam J, Thurecht KJ, Corrie SR (2016) Nanoparticle-Based Medicines: A Review of FDA-Approved Materials and Clinical Trials to Date. Pharm Res 33(10): 2373-2387.

15. Pugazhendhi A, Edison TNJ, Karuppusamy I, Kathirvel B (2018) Inorganic nanoparticles:a potential cancer therapy for human welfare. International Journal of Pharmaceutics 539(1-2): 104-111.

16. Aalinkeel R, Nair B, Chen CK, Mahajan SD, Reynolds JL, et al. (2016) Nanotherapy silencing the interleukin-8 gene produces regression of prostate cancer by inhibition of angiogenesis. Immunology 148(4): 387-406.

17. Xiaoding Xu, Jun Wu, Yanlan Liu, Phei Er Saw, Wei Tao, et al. (2017) Multifunctional Envelope-Type siRNA Delivery Nanoparticle Platform for Prostate Cancer Therapy. ACS Nano 11(3): 2618-2627.

18. Bharali DJ, Sudha T, Cui H, Mian BM, Mousa SA (2017) Anti-CD24 nanotargeted delivery of docetaxel for the treatment of prostate cancer. See comment in PubMed Commons belowNanomedicine 13(1): 263-273.

19. Orielyz Flores, Santimukul Santra, Charalambos Kaittanis, Rania Bassiouni, Amr S Khaled, et al. (2017) PSMA-Targeted TheranosticNanocarrier for Prostate Cancer. Nanomedicine 7(9): 2477-2494.

\section{Your next submission with Juniper Publishers will reach you the below assets}

- Quality Editorial service

- Swift Peer Review

- Reprints availability

- E-prints Service

- Manuscript Podcast for convenient understanding

- Global attainment for your research

- Manuscript accessibility in different formats

( Pdf, E-pub, Full Text, Audio)

- Unceasing customer service

Track the below URL for one-step submission https://juniperpublishers.com/online-submission.php 